\title{
Diagnosis and natural history of congested (dilated) cardiomyopathies
}

\author{
Celia Oakley \\ M.D., F.R.C.P., F.A.C.C. \\ Hammersmith Hospital and Royal Postgraduate Medical School, London
}

\begin{abstract}
Summary
The diagnosis of primary dilated cardiomyopathy depends on the recognition of a dilated poorly contracting left ventricle with increased end-diastolic and end-systolic volumes in the absence of a detectable cause. The diagnosis is made only after exclusion both of structural heart disease and of known causes of secondary heart muscle disorder.

The natural history is still largely unknown and is probably as variable as the likely causes. The left ventricular disorder does not cause symptoms until heart failure supervenes except for occasional patients who develop an early atrial or ventricular dysrhythmia, conduction defect, chest pain or murmur of mitral regurgitation. This period of latency may be short, prolonged or even permanent since it is unlikely that all cases progess to the point of failure. A few patients recover normal or near-normal cardiac function. The interplay between high blood pressure, hypertensive heart failure and dilated cardiomyopathy is illustrated by patients who recover from heart failure to become hypertensive and vice versa and in current treatment with vasodilators and diuretics for patients at either end of the spectrum.
\end{abstract}

\section{Introduction}

Cardiomyopathy is defined as heart disease of unknown cause. This definition excludes structural heart disease as well as heart failure secondary to known causes such as amyloid or sarcoid. Cardiomyopathy is, therefore, synonymous with primary heart muscle disease and the heart muscle diseases of known cause are regarded as secondary heart muscle disorders. Once the aetiology has been established the word Cardiomyopathy will disappear from use (Goodwin and Oakley, 1972; Oakley, 1972, 1974).

Cardiomyopathies are divided into three groups dependent on the haemodynamic and angiocardiographic abnormality. This functional classification is mirrored in the histopathology by which the same three groups can be recognized by their distinctive gross, light microscopic and ultrastructural features and called by the same names (Fig. 1).

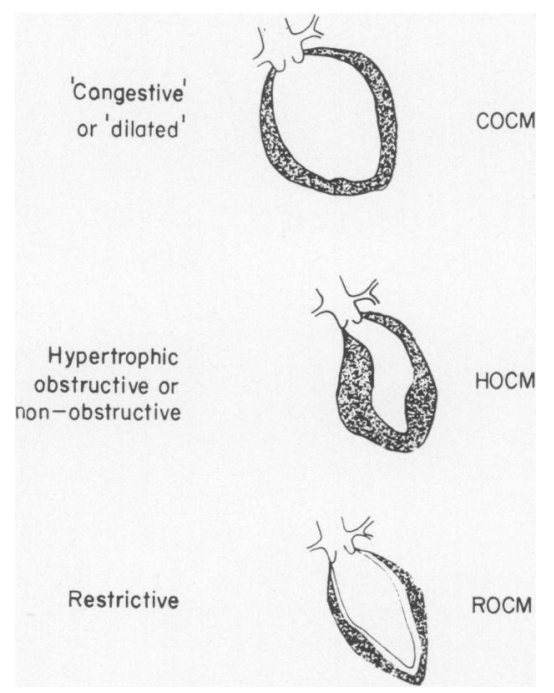

FIG. 1. The three main types of cardiomyopathy. Only in dilated cardiomyopathy is the cavity of the left ventricle enlarged. In the hypertrophic and restrictive types its dimensions are normal or small.

\section{Recognition}

The essential feature of dilated cardiomyopathy is dilatation of the cavity of the left ventricle both at end diastole and end systole. Its shape becomes more spherical as it enlarges and its walls are uniformly hypokinetic (Fig. 2). The end-diastolic pressure is usually raised. The stroke volume is normal or reduced and there may be some functional mitral regurgitation. The coronary arteries are usually completely normal. In some cases they may show atheromatous irregularity but no obstructions sufficient to account for the generalized myocardial pump failure.

\section{Case detection}

The patient most often presents with the symptoms of left ventricular failure, shortness of breath which was of gradual onset or sometimes acute. Less often, 


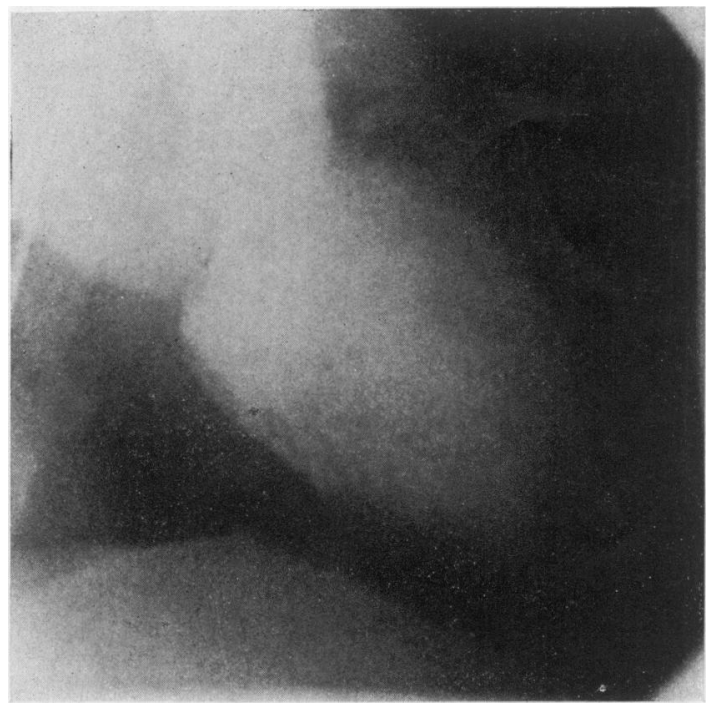

FIG. 2. The essential feature is dilatation of the left ventricle whose shape becomes more spherical as it enlarges.

the patients present on account of dysrhythmia, multiple ectopic beats, atrial fibrillation or ventricular tachycardia. Angina, which is present in about $10 \%$, may cause confusion with coronary disease. Cases may be discovered on account of a routine ECG which shows an abnormality such as left bundle branch block or because of a routine X-ray which shows an increase in heart size (Fig. 3). Patients with arrhythmia, ECG or X-ray abnormality tend to present earlier than those patients who complain of the symptoms of heart failure but the earliest manifestations and the time course of this disorder remain unknown. It is possible that there may be a long period of latency in many individuals in whom but for the development of dysrhythmia the cardiac disorder might otherwise have remained occult for some years. Probably the disorder exists in milder form and undetected in a number of individuals who never get diagnosed at all and in whom the cardiac disorder may not even shorten life. The size of this submerged iceberg remains unknown and the evidence for it is mainly circumstantial and based on the fortuitous detection of asymptomatic individuals who can subsequently be followed. A further problem which has hampered progress is the reluctance of physicians to investigate asymptomatic individuals even though they show clinical stigmata of a cardiac fault. Moreover, when such patients are investigated the abnormality in left ventricular contraction is much milder than exists in the symptomatic patient with heart failure and differences of opinion then arise as to whether

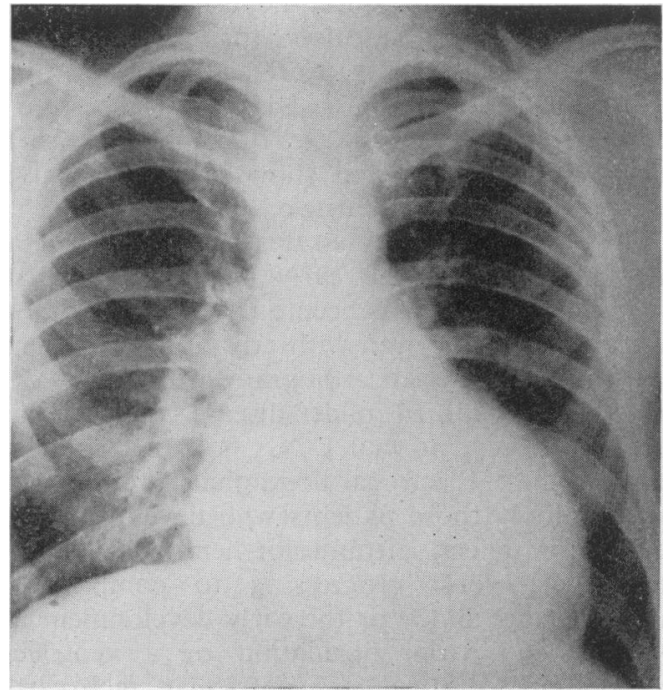

FIG. 3. The commonest presenting symptoms are related to left ventricular failure. This may be picked up on a chest radiograph taken before the cardiac source of the symptoms is recognized. This radiograph shows considerable left ventricular enlargement and denotes severe advanced disease with a poor ultimate prognosis.

a mild though definite impoverishment of left ventricular contraction should be categorized as dilated cardiomyopathy.

\section{Recognition of early disease}

A normal chest X-ray may hide considerable left ventricular dilatation provided the left atrial pressure has remained normal so that there are no pulmonary venous congestive changes. It seems that increasing deterioration of left ventricular contraction may for some time be compensated for by increasing left ventricular size at both end diastole and end systole while the difference, the stroke volume, remains normal and the filling pressure also remains normal. This does not make for early diagnosis since such patients are asymptomatic up to a very considerable degree of left ventricular dilatation. In other instances, the left ventricle may not dilate without accompanying increases in filling pressure so that the individual reports with breathlessness or with a recognizably abnormal chest X-ray at an earlier stage of dilatation; and such patients are the most likely to respond favourably to treatment. Radiologically detectable right atrial and right ventricular dilatations do not usually appear in the absence of overt left ventricular failure. This means that the routine chest X-ray is an insensitive method of detecting really early asymptomatic cases of dilated cardiomyopathy. Moreover, readers of routine chest $\mathrm{X}$-rays usually do not remark on minor 
cardiac enlargement since the usual reason for a routine chest $\mathrm{X}$-ray is to look for lung disease. The patient illustrated in Fig. 3 presented with advanced cardiomegaly and failure. His symptoms were of recent origin but a chest X-ray taken 2 years earlier and regarded as normal showed unequivocal enlargement of the left ventricle.

The ECG is similarly an unreliable and generally insensitive tool in the early detection of dilated cardiomyopathy. It has been our experience that the occasional patient even with advanced disease may still show an electrocardiogram which would be passed as normal. In milder disease or earlier cases the incidence of normal ECGs is likely to be very much higher. Electrocardiographic screening may pick out only those patients who develop an early conduction defect, left anterior hemiblock or interventricular block progressing to complete left bundle branch block or the early development of a dysrhythmia. Atrial fibrillation or a ventricular dysrhythmia, particularly ventricular tachycardia, may reveal the patient's illness at an early stage. In other cases, a systolic murmur of mitral regurgitation may be the earliest finding and is the signal to underlying left ventricular disease (Fig. 4). It could be argued that such patients may not have the same condition as those without dysrhythmia or conduction defects.

Despite these limitations, any attempt at population screening to detect early dilated cardiomyopathy would have to rely heavily upon both the chest X-ray and the ECG. It is quite uncertain what proportion of the cases would be picked up by such means and what proportion would be missed. Even full clinical examination and non-invasive studies with echo cardiography and systolic time intervals would be unlikely to reveal all the milder cases.

\section{The role of virus infection in the causation and natural history of dilated cardiomyopathy}

In the absence of an explanation for the development of left ventricular failure it was inevitable that virus infection would be suggested as a likely aetiological agent. The relation between infection with a number of known viruses and acute myopericarditis is established. Acute myopericarditis is more common in childhood, particularly infancy, than in adults and virus has been recovered from the myocardium of fatal cases. The problem is that milder cases are far more common and, in these, the diagnosis of virus myocarditis is largely circumstantial. In a few cases seen early, virus may be recovered from throat washings or from stool but more often by the time that a cardiac disorder has been suspected, throat and stool cultures are negative and reliance has to be placed on rising or falling titres together with clinical phenomena. Probably more

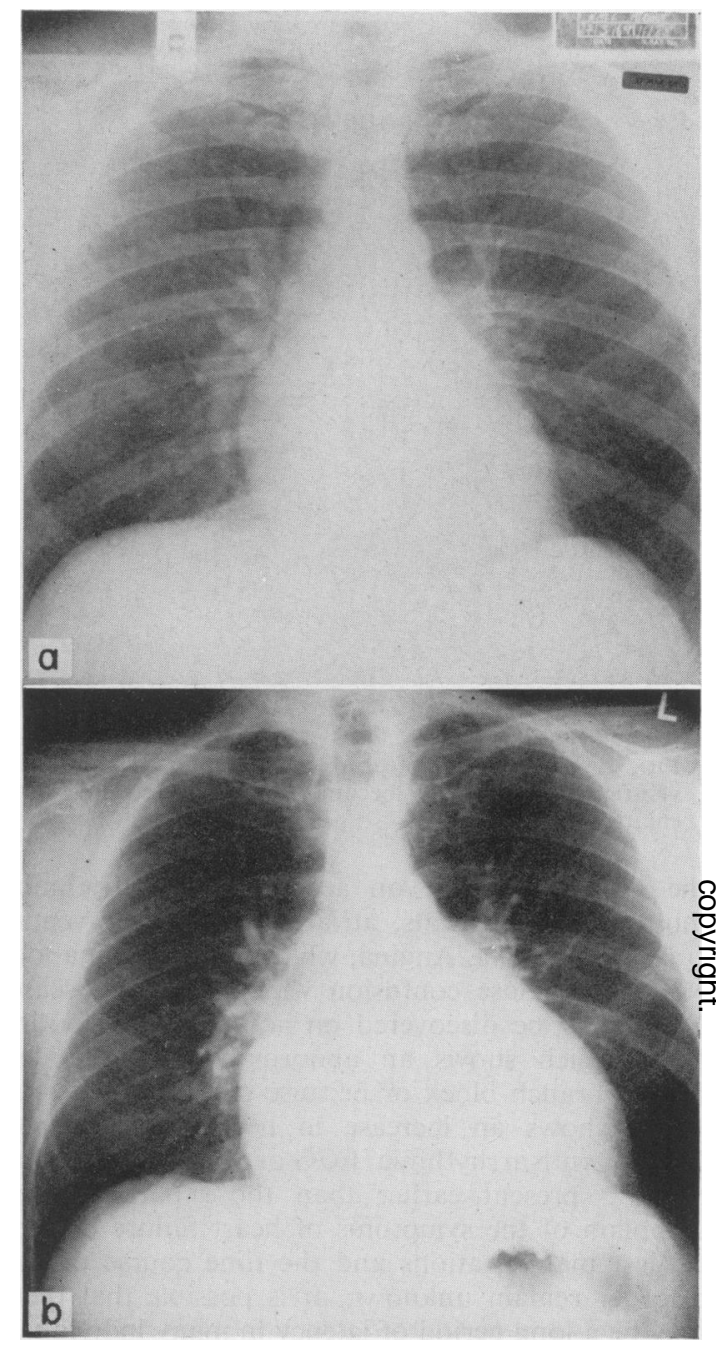

Fig. 4. This patient presented with atrial fibrillation and a pansystolic murmur at the apex. He was found to have a dilated poorly contracting left ventricle with mild mitral reflux (a). Now 4 years later he remains asymptomatic despite the huge further increase in heart size (b). Much of this increase in size of the cardiac shadow is due to distension of the fibrillating left atrium but the left ventricle has also enlarged further.

cases are never diagnosed at all than are investigated at the time of an acute virus illness and so the link between the established acute virus myocarditis and dilated cardiomyopathy has never been established.

The possible role of virus infection is further clouded by the tendency of doctors seeing a previously vigorous patient presenting for the first time with cardiac symptoms to diagnose a virus infection rather than to recognize the patient's first episode of 
left ventricular failure for what it is. Fig. 3 exemplifies this. Virus infection may, therefore, act as a trigger or simply be over-emphasized because of medical anxiety to find an acceptable cause of a previously healthy patient's unexpected illness.

Most patients who survive acute myocarditis seem to recover completely but if virus myocarditis is often sub-clinical then neutralizing antibody titres might remain high for long enough after infection to be detected in patients presenting with the chronic disorder. Furthermore, although cardiac biopsies are rarely possible during acute myocarditis, biopsy material can be obtained from patients being investigated on account of chronic left ventricular disorder (Fig. 5). Investigation of fifty patients with dilated cardiomyopathy and fifty age- and sex-matched control patients was carried out at the Royal Postgraduate Medical School (Cambridge et al., 1978). Patients with dilated cardiomyopathies did indeed show a higher incidence of titres in excess of $1 / 1024$ against Coxsackie $B$ virus. In addition, it emerged that these very high titres were commoner in patients with a short history of illness than in those with a longer history. This association does not prove causation: the patients might have had chronic myocarditis or the rise in antibody titres might have been hetero-typical. The most appealing explanation for the observation, however, is that virus infection might have 'exposed' sub-clinical left ventricular dysfunction in individuals who might not otherwise have developed a symptomatic abnormality or come to medical attention.

The role of chronic alcohol abuse in the causation and natural history of dilated cardiomyopathy

The same arguments can be used to link excessive alcohol consumption with dilated cardiomyopathy

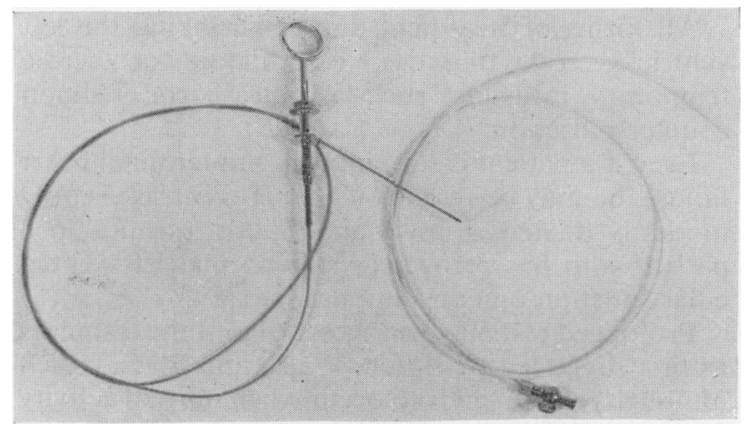

FIG. 5. Olympus bronchoscopic bioptome which is passed through a radio-opaque catheter sheath. The catheter is first introduced via a femoral vein Seldinger puncture and the tip left at the chosen site in the right ventricle. Multiple biopsies can be taken from different points using the catheter conduit for rapid access. although alcoholic cardiomyopathy is a timehonoured concept (Brigden and Robinson, 1964). Alcohol is a myocardial depressant agent but many other substances also depress the mycoardium while in the body tissues. The acute myocardial depression induced by alcohol infusion and associated with measured high blood levels of alcohol does not prove that alcoholism causes dilated cardiomyopathy. Similarly, improvement after withdrawal of alcohol offers no proof of causation. The left ventricular fault and the histopathological abnormalities are no different in the alcoholic patient from the nonalcoholic patient and the suggestion of some small differences in ultrastructure or in myocardial enzyme concentrations could also result from the acute potentially transient effect of alcohol rather than be an indication of its causal role. (A suitable analogy would be with aldactone bodies seen in the kidneys of patients taking spironolactone.) More relevant would be the demonstration of morphological or biochemical differences in the myocardium of patients with cardiomyopathy who had been alcoholic but from whom alcohol had been withdrawn some time previously.

A further difficulty in the link between alcohol and dilated cardiomyopathy lies in the infrequency of cardiac disorder in patients with cirrhosis of the liver of presumed alcoholic origin.

It seems most likely, therefore, that alcohol may not work as a causative agent in its own right but as a malevolent influence whereby mild or subclinical abnormality is brought to light by the depressant effect of alcohol upon the already ailing myocardium. Conversely, alcohol may not be a cause of chronic progressive left ventricular failure in those whose myocardium is not already underprivileged. Alcoholism cannot, therefore, be regarded as a 'risk factor' for dilated cardiomyopathy in the way that smoking is a 'risk factor' in a patient with possible angina. Nevertheless, the malevolent influence of alcohol upon the abnormal myocardium is undoubted and alcoholism may have an unfavourable influence upon the natural history of dilated cardiomyopathy.

It is proper to advise a patient with dilated cardiomyopathy against taking alcohol since even one or two drinks have been shown to exert a detectable effect on left ventricular performance when tested by systolic time intervals and echo cardiographicallydetermined parameters of left ventricular efficiency.

The role of high blood pressure upon the development and natural history of dilated cardiomyopathy

The observation that raised blood pressure levels are commonly recorded in patients with left ventricular failure and diagnosed as having dilated cardiomyopathy led to an interest in the possible relation 
between high blood pressure and the development and natural history of the disorder. It has long been known that blood pressure may rise during acute left ventricular failure, owing, presumably, to excessive catecholamine-induced vaso-constriction. Subsequent improvement associated with diuretic treatment is usually accompanied by a fall in blood pressure to normal levels. The difficulty in establishing the role of high blood pressure is compounded by the fact that diuretics used for treating heart failure are effective blood pressure lowering agents in hypertensives without heart failure. Given a patient with heart failure due to any cause, the calculated peripheral vascular resistance (or, more correctly, the impedance) is well above normal if the cardiac output is low. Otherwise, normal blood pressure would not be maintained in heart failure. If that raised peripheral vascular impedance remains unchanged and the patient recovers from heart failure, then the blood pressure rises and the diagnosis is likely to be 'hypertension'. In other words the 'diagnosis' varies between dilated cardiomyopathy and hypertension according to left ventricular function and only if a patient with dilated cardiomyopathy, heart failure and normal blood pressure actually recovers and develops high blood pressure when not in heart failure can the causal or conditioning role of high blood pressure be proved. Sometimes we see this phenomenon. Much more often patients with dilated cardiomyopathy do not recover normal left ventricular function, either with or without hypertension. The development of left ventricular failure because of hypertension determines a fall of the previously raised blood pressure to normal levels and, since the failure usually persists, the blood pressure remains normal. Since the vascular damage is caused by sustained high blood pressure, then patients with dilated cardiomyopathy caused by hypertension may not show the vascular stigmata of hypertension which can be recognized in chronically hypertensive patients. It seems possible that left ventricular failure may be more likely to develop in a hypertensive when the blood pressure rise has been rapid than when it has been of slow development.

The left ventricular wall thickness is highly variable in patients who have died with the diagnosis of dilated cardiomyopathy. Although heart weights are increased in all, the thickness of the left ventricular wall may be normal when this chamber is very dilated and this is because of myocardial fibre slippage even though the left ventricles are truly hypertrophied. In other patients, the left ventricular wall thickness may be markedly increased despite a similar amount of left ventricular dilatation. This seems to be a particular feature of cardiomyopathy in Africa and the massive hypertrophy which these hearts often show led to the condition being called 'idiopathic cardiomegaly' by workers in Kampala $\frac{2}{\widetilde{\alpha}}$ and Ibadan. In black Africa, heart muscle disorder $\cong$ of this sort accounts for $40 \%$ or more of all heart $\stackrel{c}{\Rightarrow}$ disease seen, despite high incidences of rheumatic $\vec{F}$ and infective valve disease. In these countries, $\overrightarrow{0}$ coronary disease remains rare but hypertension is $\frac{C}{0}$ exceedingly common. The appearances of the hearts $\frac{\overline{\bar{\omega}}}{2}$ of patients who have died in chronic left ventricular failure associated either with well documented $\cong$ previous hypertension or with idiopathic cardio- $\infty$ megaly and normal blood pressure are indistinguish- $\overrightarrow{0}$ able. In the West, where high blood pressure is more often detected and treated, death from stroke or left $\vec{\sigma}$ ventricular failure is rare and myocardial infarction? is the commonest cause of death in patients in this 3 country with treated hypertension. This problem if does not complicate the issue in the African coun- + tries, and Brockington (personal communication) commented on the treatment of patients with left ${ }^{\omega}$ ventricular failure in Nigeria in some of whom im- $+\overrightarrow{+}$ provement in left ventricular function is associated with a rising blood pressure and re-establishment of ${ }^{\circ}$ hypertension despite the use of diuretics which $\overrightarrow{\vec{c}}$ normally lower blood pressure in uncomplicated hypertension.

A role for hypertension in the genesis of dilated $\vec{e}$ cardiomyopathy seems very likely but the proportion 90 of patients in whom previous hypertension has be the major or only factor in causing left ventriculär failure remains unknown. In other cases, such as those of virus infection and those affected by alcohol, high blood pressure may play only a supple- $\frac{\mathbb{Q}}{2}$ mentary role in precipitating failure in a heart which $\overrightarrow{\overrightarrow{0}}$ has been damaged by other agents still unknown. Treatment and the relevance of left ventricular cavityo
size to prognosis

Once the left ventricle has become very distended 3 its function never returns to normal.

All forms of treatment aim at shrinking the left ventricle; rest, diuretics, vasodilators or heroic temporary measures such as intra-aortic balloon counter pulsation.

Even if a patient is seemingly in pre-terminal heart $\frac{\text { ? }}{2}$ failure, he may be rescued if the left ventricle is only은 modestly dilated. Figs. 6 and 7 illustrate this in a patient who has recovered near normal left ventri- $\sigma$ cular function on conventional therapy.

Prolonged rest may be successful in some instances N as first suggested by Burch, Walsh and Black (1963) ${ }_{\sigma}^{\omega}$ although relapse tends to occur when normal activity is resumed (Fig. 8).

\section{The role of cardiac biopsy}

Myocardial biopsy is now a safe and well estab- $\frac{0}{0}$ lished procedure when carried out after the manner市 first described by Sakakibara and Konno in $1962 \frac{\mathrm{\rho}}{\mathrm{Q}}$ 

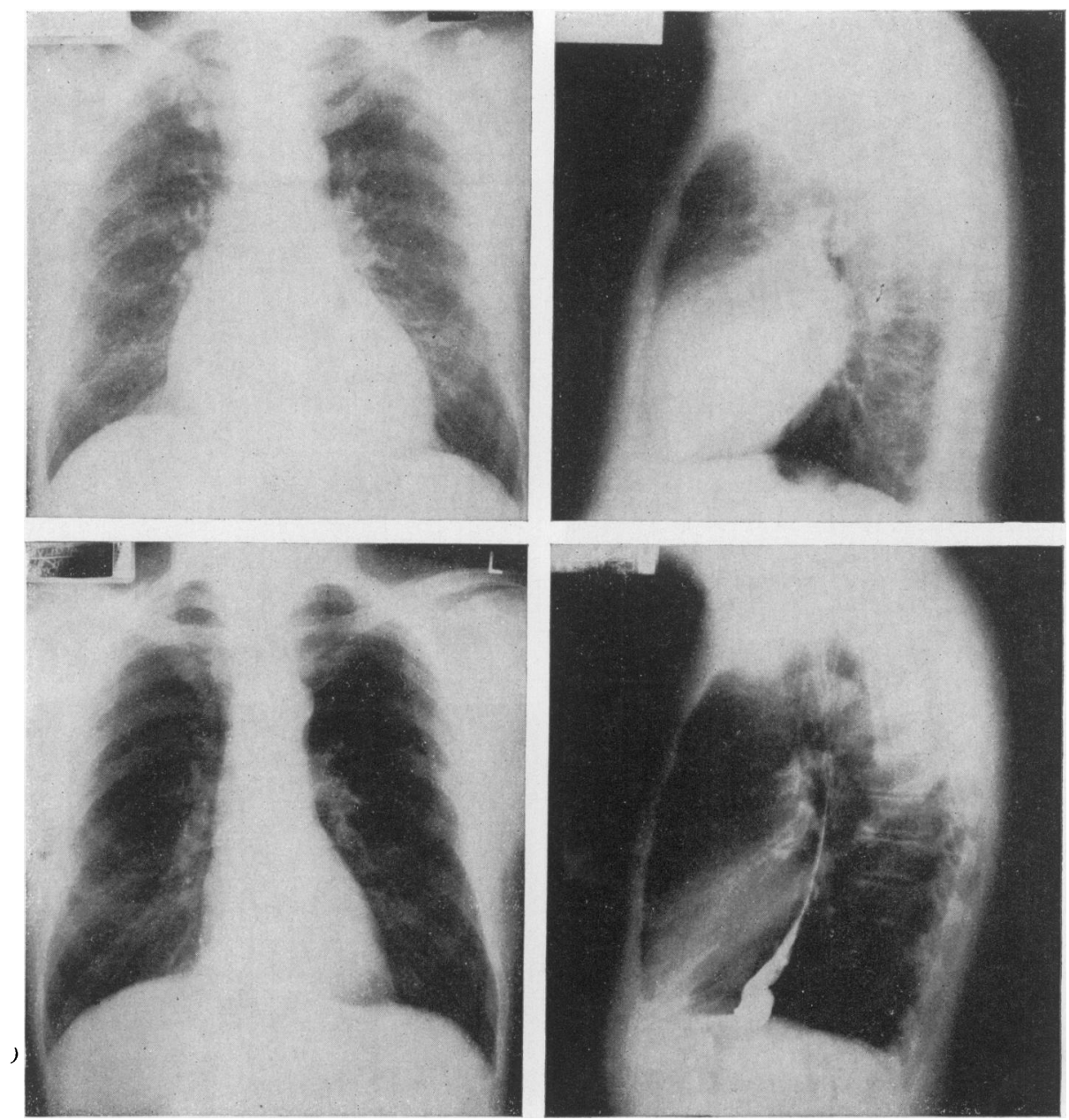

FIG. 6. Plain chest radiography from a patient who has recovered virtually normal left ventricular function after 2 years of conventional anti-failure treatment. At the top is the presenting film when he was in low output congestive failure; and at the bottom is the current film as he is today, asymptomatic and no therapy. The diminution in heart size is well seen in both the PA and lateral projections.

and plays an important role in excluding heart muscle disorder due to known agents such as haemochromatosis or sarcoidosis even though these disorders are not usually occult in patients presenting with features of dilated cardiomyopathy. With further refinement of the techniques of examination, the biopsy specimens are begining to reveal some correlation between the amount of morphological derangement of the ultrastructure and the natural history and prognosis of the disorder. Continuing intensive study of the morphological and biochemical derangement in the heart muscles of patients will surely lead ultimately to an understanding of the pathogenesis of the myocardial failure (Peters et al., 1977).

\section{Dilated cardiomyopathy, one disease or many?}

Almost certainly many disorders lead towards the final common pathway of chronic left ventricular pump failure and by the time we see the patient we are looking at the end-result. Recognizing that a murder has been committed does not necessarily help to identify the criminal. Only a painstaking scrutiny of all possible clues with cross-examination and 
Fig. 7. Left ventricular angiograms from the same patient as in Fig. 6 (end-systole on the left and end-diastole on the right). At the top is the poorly contracting left ventricle as it was and at the bottom the near-normal left ventricle as it is today. The change in shape from near-splenial when in failure to a slim ellipsoid and recovery is also well seen as is the mitral regurgitation which was present when he was in left ventricular failure.

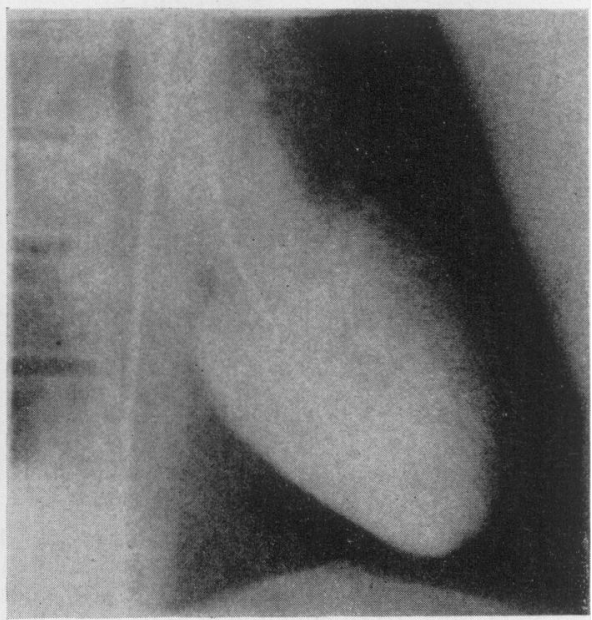

옥

$\frac{D}{0}$

量.

N

ก

N

ర

$\stackrel{2}{\infty}$ 

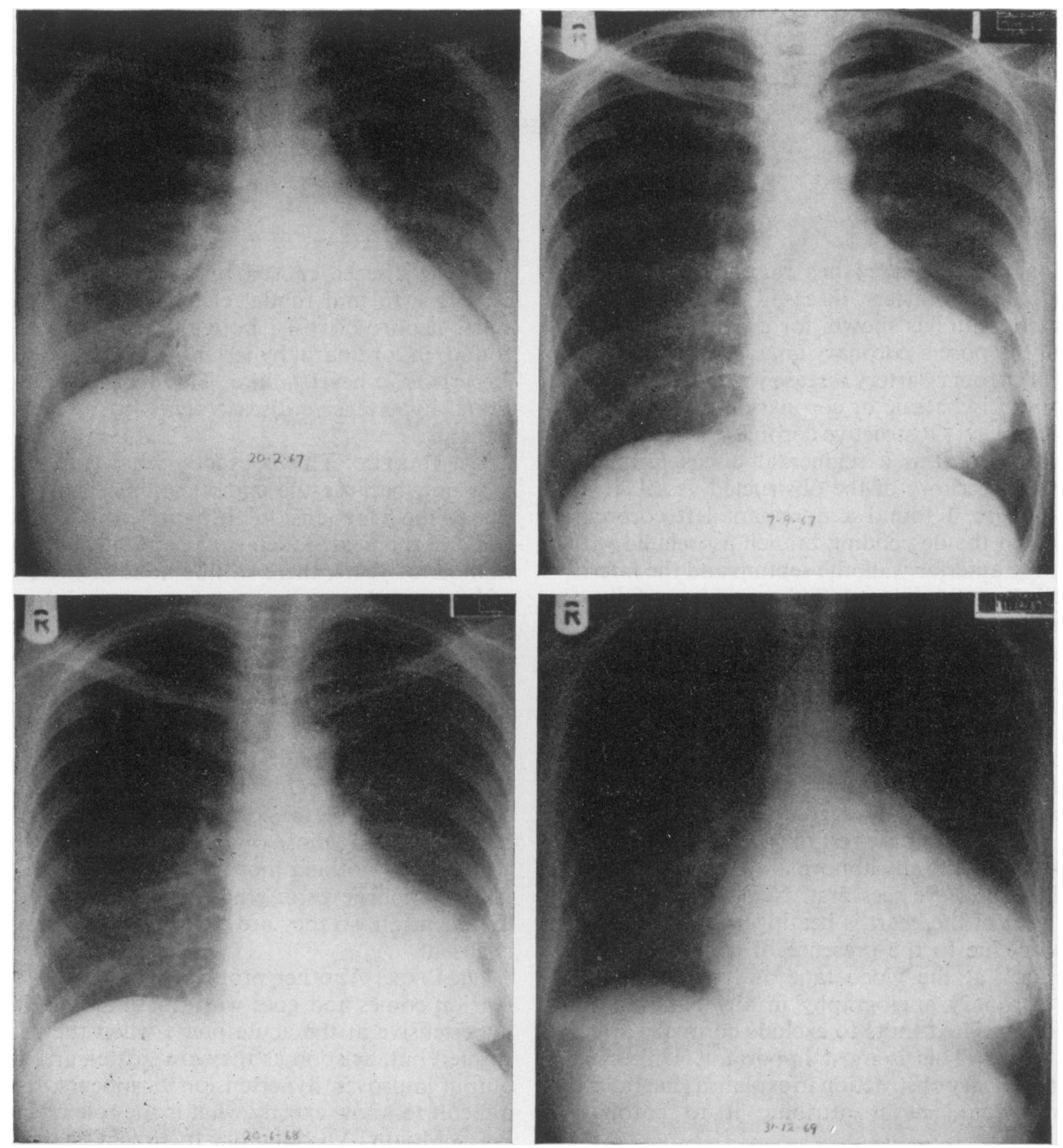

FIG. 8. The effects of rest on heart failure and heart size are well shown in this patient but deterioration occurred when she recovered normal activity.

gradual elimination of the suspects leads to a conviction - and that is what we have to do in our research to conquer the enigma that is dilated cardiomyopathy.

\section{References}

Brigden, W.W. \& Robinson, J.F. (1964) Alcoholic heart disease. British Medical Journal, 2, 1283.

Burch, G.E., Walsh, A. \& Black, W.L. (1963) Value of prolonged bed rest in the management of cardiomegaly. Journal of the American Medical Association, 183, 81.

Cambridge, G., MacArthur, C.G.C., Waterson, A.P.,
Goodwin, J.F. \& OAKLey, C.M. Antibodies to Coxsackie B viruses in primary congestive cardiomyopathy. New England Journal of Medicine, (In press).

Goodwin, J.F. \& OAKLEY, C.M. (1972) The cardiomyopathies. British Heart Journal, 34, 545.

OAKLEY, C.M. (1972) Clinical definition and classification of cardiomyopathies. Postgraduate Medical Journal, 48, 703.

OAKLEY, C.M. (1974) Clinical definition of the cardiomyopathies. Circulation Research, 35 (Suppl.), 152.

Peters, T. J., Wells, G., OAKley, C.M., Brooksby, I.A.B., Jenkins, B.S., WebB-PePloe, M.M. \& ColtarT, D.J. (1977) Enzymic analysis of endomyocardial biopsy specimens from patients with cardiomyopathies. British Heart Journal, 39, 1333.

SaKakibara, S. \& Konno, S. (1962) Endomyocardial biopsy. Japanese Heart Journal, 3, 537. 